\title{
RELIABILITY OF BYSTANDER RECOGNITION OF CliniCAL FEATURES IN PRE-HOSPITAL CLASSIFICATION OF ACUTE CEREBROVASCULAR SYNDROMES: PRELIMINARY FINDINGS
}

\author{
Erwin Chiquete ${ }^{1 *}$, Valeria Sandoval-Rodríguez ${ }^{1}$, Miguel García-Grimshaw ${ }^{1}$, Amado Jiménez-Ruiz ${ }^{1}$, \\ Juan J. Gómez-Piña ${ }^{1}$, Eduardo Ruiz-Ruiz ${ }^{1}$, Guillermo Ramírez-García ${ }^{1}$, Fernando Flores-Silva ${ }^{1}$, \\ Carlos Cantú-Brito ${ }^{1}$, Ana Ochoa-Guzmán ${ }^{2}$, and José L. RuIz-Sandoval 3,4 \\ ${ }^{1}$ Department of Neurology and Psychiatry and ${ }^{2}$ Molecular Biology Unit, Instituto Nacional de Ciencias Médicas \\ y Nutrición Salvador Zubirán, Mexico City; ${ }^{3}$ Neurology Service, Hospital Civil de Guadalajara Fray Antonio Alcalde, \\ Guadalajara, Jal.; ${ }^{4}$ Department of Neuroscience, Centro Universitario de Ciencias de la Salud, Universidad de \\ Guadalajara, Guadalajara, Jal., Mexico
}

\begin{abstract}
Background: The recognition of stroke symptoms by patients or bystanders directly affects the outcomes of patients with acute cerebrovascular disease. Objective: The objective of the study was to assess the predictive value of the medical history and clinical features recognized by the patients' bystanders to classify neurovascular syndromes in pre-hospital settings. Methods: We included 150 stroke patients of two Mexican referral centers: 50 with acute ischemic stroke (AIS), 50 with intracerebral hemorrhage $(\mathrm{ICH})$, and 50 with subarachnoid hemorrhage $(\mathrm{SAH})$. The performance of clinical prediction rules (CPR) to identify the stroke types was evaluated with features recognized by the patients' bystanders before hospital arrival. The impact of CPRs on early arrival and in-hospital mortality was also analyzed. Results: Overall, $72 \%$ of the patients had previous medical evaluations in other centers before final referral to our hospitals, and therefore, only $45 \%$ had a final onsetto-door time $<6 \mathrm{~h}$, even when the first medical assessment had occurred in $\leq 1 \mathrm{~h}$ in $75 \%$ of cases. Clinical features noticed by the patients' bystanders had low positive predictive values (PPV) for any stroke type. The CPR "language or speech disorder + focal motor deficit" had $93 \%$ sensitivity and a negative predictive value (NPV) of $84 \%$ to distinguish AIS. In SAH, headache alone showed a sensitivity of $84 \%$ and NPV of $97 \%$. No CPR had an adequate performance on ICH. CPRs were not associated with final onset-to-door time. Altered consciousness, age $\geq 65$ years, indirect arrival with stops before final referral, and atrial fibrillation increased in-hospital mortality. Conclusion: Clinical features referred by the witness of a neurovascular emergency have limited PPV, but adequate NPV in ruling-out AIS and SAH among stroke types. The use of CPRs had no impact on onset-to-door time or in-hospital mortality when the final arrival to a third-level center occurs with previous medical referrals. (REV INVEST CLIN. 2021;73(2):87-93)
\end{abstract}

Key words: Acute ischemic stroke. Clinical manifestations. Intracerebral hemorrhage. Prognosis. Subarachnoid hemorrhage.

*Corresponding author:

Erwin Chiquete

E-mail: erwin.chiquetea@incmnsz.mx
Received for publication: 23-05-2020

Approved for publication: 26-08-2020

DOI: $10.24875 /$ RIC.20000238

0034-8376 / (c) 2020 Revista de Investigación Clínica. Published by Permanyer. This is an open access article under the CC BY-NC-ND license (http://creativecommons.org/licenses/by-nc-nd/4.0/). 


\section{INTRODUCTION}

Initial and timely recognition of an acute stroke by the event's witness is a factor that directly affects the outcome and functional prognosis in all neurovascular emergencies ${ }^{1-4}$. The pre-hospital classification of other vascular emergencies, such as acute coronary syndrome, has proven to shorten the response time by the emergency medical system (EMS), improving the short-term prognosis ${ }^{5,6}$. In ischemic stroke, a short time from symptom onset to clinical assessment makes patients eligible for reperfusion therapies ${ }^{7-9}$. In the setting of a neurovascular emergency, the preclassification of the stroke type by the EMS may reduce the time to treatment, which may improve clinical outcomes.

The primary objective of the present study was to investigate the predictive value of the patients' past medical history and early clinical features, recognized by a subject witnessing the event, to be used in the pre-hospital classification of the most common stroke types (e.g., ischemic, hemorrhagic, or subarachnoid hemorrhage $[\mathrm{SAH}]$ ), according to subsequent neuroimaging studies. With this pre-hospital information (i.e., past medical history and clinical features recognized by witnesses), we created clinical prediction rules (CPRs). Thus, a secondary objective was to determine the possible impact of CPRs on the outcome at discharge. With these analyses, we explored the feasibility of a pre-hospital classification of stroke types before ambulance dispatch and its impact on short-term outcome.

\section{METHODS}

In this prospective study, we included patients treated at the Hospital Civil de Guadalajara Fray Antonio Alcalde (Guadalajara, Jalisco) $(n=99)$ and at the Instituto Nacional de Ciencias Médicas y Nutrición Salvador Zubirán (Mexico City) $(n=51)$ with a confirmed neuroimaging diagnosis as the gold standard (computed tomography or magnetic resonance imaging) of acute ischemic stroke (AIS), intracerebral hemorrhage $(\mathrm{ICH})$, and $\mathrm{SAH}$. The local ethics committees of both hospitals approved the protocol. We randomly selected 50 cases of each of the three included stroke types (AIS, $\mathrm{ICH}$, or $\mathrm{SAH}$ ) from a larger prospective database on stroke patients, excluding cases of transient ischemic attack or cerebral venous thrombosis. These case records were selected among patients hospitalized in each center from January 2011 to December 2015. Although the proportions of stroke types differ (AIS being the most common), the random selection of stroke syndromes was performed unbiased at an equal quota to balance the statistical analyses on the prediction of stroke types using the bystanders' reference. The stroke onset was defined by the time the patient was last seen to be well, as referred by the subject who witnessing the event. The time of admission was considered as the time of arrival registered in the emergency department (ED). An indirect arrival (with stops or detours) was considered as any arrival to a first-aid service, clinic, or hospital before arriving at our center. Cases in which the onset of clinical manifestations could not be accurately determined, those in which the stroke occurred during sleep or during any hospital stay, and cases with incomplete information on the clinical records were also excluded from the study. We collected the data with a standardized structured case report format (CRF) specifically created for the purposes of the study to be used in a homogeneous and standard fashion by a total of two trained researchers, one per participating center. This CRF has been previously used in other multicenter research projects in Mexi$\mathrm{Co}^{10}$ and included the patient's demographic characteristics, past medical history, and pre-hospital clinical manifestations (gathered from the subject who witnessed the event). The clinical features were grouped into the pre-established neurovascular categories. The data were collected by interviewing directly the patient, the patient's family, or any other proxy who witnessed the onset of the neurovascular syndrome.

Clinical evaluations reported by attending physicians were excluded, and only the data provided by the witness was analyzed to emulate a telephone interview between a member of the EMS and the initial witness of the event. We performed this task to assess whether the results of a pre-hospital classification could be easily applied by EMS personnel to identify the stroke subtype before the ambulance dispatch or arrival of the patient to the ED. At present (and at the time the study was carried out), there is no telephone triage in Mexico done by EMS to pre-classify a neurovascular syndrome. Level of consciousness was classified using the bystanders' reference on the patients' behavior as follows: alert, lethargy, somnolence, 
Table 1. Baseline characteristics of the 150 patients included in the study

\begin{tabular}{|c|c|c|c|c|}
\hline \multirow[t]{2}{*}{ Characteristics } & \multicolumn{3}{|c|}{ Stroke type } & \multirow[t]{2}{*}{ p-value* } \\
\hline & AIS $(n=50)$ & $\mathrm{ICH}(n=50)$ & SAH $(n=50)$ & \\
\hline Age, years, median (range) & $68(25-87)$ & $58(28-98)$ & $58(22-77)$ & 0.24 \\
\hline Female, sex, n (\%) & $32(64.0)$ & $21(42.0)$ & $31(62)$ & 0.05 \\
\hline $\begin{array}{l}\text { Educational level, } \geq 6 \text { years, } \\
\text { n (\%) }\end{array}$ & $37(74.0)$ & $43(86.0)$ & $47(94.0)$ & 0.02 \\
\hline \multicolumn{5}{|l|}{ Risk factors } \\
\hline Arterial hypertension, n (\%) & $27(54.0)$ & $38(74.0)$ & $26(52.0)$ & 0.02 \\
\hline Obesity, n (\%) & $21(42.0)$ & $23(46.0)$ & $34(68.0)$ & 0.02 \\
\hline Diabetes mellitus, n (\%) & $14(28.0)$ & $15(30.0)$ & $10(20.0)$ & 0.48 \\
\hline Previous stroke, n (\%) & $12(24.0)$ & $11(22.0)$ & $2(4.0)$ & 0.02 \\
\hline Atrial fibrillation, $\mathrm{n}(\%)$ & $9(18.0)$ & $3(6.0)$ & $1(2.0)$ & 0.01 \\
\hline \multicolumn{5}{|l|}{ Clinical features } \\
\hline $\begin{array}{l}\text { Language or speech disorder, } \\
\mathrm{n}(\%)\end{array}$ & $49(98.0)$ & $42(84.0)$ & $29(58.0)$ & $<0.001$ \\
\hline Focal motor deficit, $\mathrm{n}(\%)$ & $47(94.0)$ & $46(92.0)$ & $32(64.0)$ & $<0.001$ \\
\hline Headache, n (\%) & $18(36.0)$ & $18(36.0)$ & $41(82.0)$ & $<0.001$ \\
\hline Vomiting, n (\%) & $9(18.0)$ & $20(40.0)$ & $36(72.0)$ & $<0.001$ \\
\hline $\begin{array}{l}\text { Altered level of consciousness, } \\
\mathrm{n}(\%)\end{array}$ & $7(14.0)$ & $20(40.0)$ & $32(64.0)$ & $<0.001$ \\
\hline
\end{tabular}

*Comparison between stroke types was performed with $\chi^{2}$ and Kruskal-Wallis test. AIS: acute ischemic stroke; ICH: intracerebral hemorrhage; $\mathrm{SAH}$ : subarachnoid hemorrhage.

stupor, and coma. An altered level of consciousness was considered any state different to alert (i.e., normal awake and response state). Final onset-to-door time was defined as the time from stroke symptoms onset (or the time of "last seen well") to final hospital arrival to our referral centers.

Demographic data, risk factors, and clinical characteristics are presented as measures of central tendency. Analyses of differences between categorical variables were performed with the Fisher $\chi^{2}$ test, as for the non-parametric continuous variables, the MannWhitney U-test, and the Kruskal-Wallis test were used. The Spearman rho correlation was used in the continuous association between two variables. To find prediction variables between arrival time and hospital mortality, we conducted a binary logistic regression, including the independent co-variables with a $\mathrm{p} \leq 0.1$. Odds ratios (OR) with $95 \%$ confidence intervals $(\mathrm{Cl})$ were calculated. The model adjustment was evaluated by the Hosmer-Lemeshow goodness of fit test, which was considered as reliable when $p \geq 0.20$. We calculated sensitivity, specificity, positive predictive value (PPV), and negative predictive value (NPV) to evaluate different clinical models for distinguishing between stroke types. Since the time for hospital arrival and the in-hospital mortality are multicausal dependent variables, only the results of multivariate analysis are considered in the explanation of cause and effect phenomena, which is why they are the only ones discussed here. All values were two-tailed and considered significant when the $p \leq 0.05$. Statistical package SPSS v24.0 (IBM; USA) and EBM v1.2 (Toronto, Ontario; Canada) were used for all calculations.

\section{RESULTS}

The study included 150 patients with acute neurovascular syndromes ( 69 men and 81 women), with a median age of 62 years (range: 21-98 years) (Table 1 ). There were no significant differences between the two 
Table 2. Multivariate analysis of factors associated with in-hospital mortality

\begin{tabular}{lcc}
\hline Variable & OR 95\% Cl & p-value* \\
\hline Age $\geq 65$ years & $3.98(1.38-10.09)$ & 0.01 \\
Indirect arrival with previous stops & $5.77(1.35-22.13)$ & 0.02 \\
Altered level of consciousness & $10.58(3.38-31.39)$ & $<0.001$ \\
Atrial fibrillation & $12.49(1.78-77.15)$ & 0.01 \\
\hline
\end{tabular}

*Model adjusted for sex, education level, past medical history, and clinical manifestations (Hosmer-Lemeshow goodness of fit test: $\chi^{2}: 5.4,5 \mathrm{df}, p=0.59$ ).

Only variables significantly associated with the final model are included in the table.

$\mathrm{Cl}$ : confidence interval.

OR: Odds ratios.

participating centers concerning clinical features, critical times of medical evaluations and arrivals, or outcomes. None of the patients studied were referred to another institution after final arrival to our two participating centers. In all, $72 \%$ of the patients had at least one previous medical evaluation in other centers before final referral to our hospitals, and as a consequence, only $28 \%$ of the patients had a direct arrival to our centers without a previous referral from another hospital: 14 (28\%) with AIS, 15 (30\%) with ICH, and 13 (26\%) with SAH. Nonetheless, 113 (75.3\%) patients had their first medical evaluation in $<1 \mathrm{~h}$, which indicates a generally fast reaction by the patients' bystander. The first medical evaluation in $<1 \mathrm{~h}$ occurred in 40 (80\%) AIS patients, in $38(76 \%) \mathrm{ICH}$ patients, and in 35 (70\%) patients with SAH.

Only 38 (25.3\%) patients arrived to the EDs of our centers (final onset-to door time) in < $3 \mathrm{~h}$ : 18 (36\%) with AIS, 11 (22\%) with $\mathrm{ICH}$, and 9 (18\%) with SAH $(p=0.09)$. In turn, $67(44.7 \%)$ patients presented to our hospitals in $<6$ h: 27 (54\%) with AIS, 22 (44\%) with $\mathrm{ICH}$, and $18(36 \%)$ with SAH $(p=0.19)$. The most common clinical manifestation noticed by the patient's bystander was a focal motor deficit (mono or hemiparesis) in 125 (83.3\%) patients followed by language or speech disorder (aphasia or dysarthria) in 120 (80\%) patients.

Supplemental Tables 1-3 describe the performance of the past medical history, clinical features, and some clinical rules for discrimination between the studied neurovascular syndromes. Overall, we found that relying on clinical features noticed by a witness alone has a low PPV for correctly predicting a stroke. The clinical rule consisting of language or speech disorder + focal motor deficit presented high sensitivity (93.1\%) and NPV (84\%) to distinguish AIS from $\mathrm{ICH}$ and SAH
(Supplemental Table 1). For the detection of ICH, the combination of focal motor deficit + history of hypertension performed better for discrimination among stroke subtypes (sensitivity $71.6 \%$, specificity $56.2 \%$, PPV $61.3 \%$, and NPV 66.6\%). However, the separate analysis of those two variables showed better performance at the cost of lower sensitivity and PPV (Supplemental Table 2). We can observe a similar pattern for $\mathrm{SAH}$, in which headache was the most reliable symptom to distinguish among stroke subtypes (sensitivity $84.1 \%$ and specificity $63.6 \%$ ) with a low PPV (22.8\%) and high NPV (97.1\%). Headache alone was even better than the clinical rule of "headache + vomiting + an altered level of consciousness" (Supplemental Table 3).

The clinical manifestations did not influence the final onset-to-door time within the first hour. A multivariate analysis to evaluate the putative influence of the clinical features on the final onset-to-door time showed that age $\geq 65$ years (OR: $5.59,95 \% \mathrm{Cl}: 1.67-$ 17.57; $p=0.004$ ) and arrival to the hospital without detours or stops along the way (OR: $17.25,95 \% \mathrm{Cl}$ : 4.60-54.13; $p<0.001$ ) were the only factors associated with a shorter final onset-to-door time (Supplemental Table 4). Overall, in-hospital mortality (i.e., case fatality rate) was $38.7 \%$, higher for SAH (48\%) and ICH (40\%) than for AIS (28\%). The cause of death was deemed neurological in $69 \%$, and no neurological in $31 \%$, mainly sepsis ( $76 \%$ ), venous thromboembolism ( $21 \%)$, and other systemic causes (3\%). In a multivariate analysis aimed to find factors associated with in-hospital mortality, we found that age $\geq 65$ years, indirect arrival with previous stops, history of atrial fibrillation, and altered level of consciousness before arrival were directly associated with in-hospital death, regardless of the clinical features, CPRs, or stroke types (Table 2). 


\section{DISCUSSION}

The recognition of an acute stroke may vary among different populations ${ }^{10-14}$. The communication between the EMS personnel and the bystander witnessing an acute cerebrovascular emergency with preestablished methods has improved clinical outcomes in other countries ${ }^{3,12,15}$. The in-hospital outcome of stroke patients might improve by identifying a neurovascular emergency correctly, regardless of its final etiology $4,5,12,16,17$. Our objective was to determine the feasibility and impact of pre-hospital clinical rules to determine the type of stroke, to assess if CPRs could be used by the EMS personnel to implement rapid treatment strategies, especially for reperfusion therapy of AIS.

In our study, the time to a first medical evaluation in $<1 \mathrm{~h}$ occurred in $75 \%$ of patients, but only $25 \%$ arrived to our referral centers in $<3 \mathrm{~h}$. This salient discrepancy may reflect that recognition of stroke symptoms by witnesses or the patients is usually opportune, regardless the stroke type, and that in the majority of cases, they are acting rapidly seeking for medical evaluation in $<1 \mathrm{~h}$, but that this first medical assessment occurs in a medical center that is not prepared to treat stroke patients, ultimately referring the patients to a dedicated center, and thus, wasting critical time to establish appropriate medical care. This unfortunate fact may be due to a reduced sensitivity of the medical staff or an inadequate hospital infrastructure ${ }^{11,18,19}$. In the $\mathrm{ICH}$ and SAH cases, we detected a lower final onset-to-door time, probably because the clinical presentation of these stroke types is usually perceived as more severe $^{11,12}$. We also found that a direct arrival (without detours in other medical institutions) and having an age of 65 years or older are factors associated with a shorter final onset-to-door time and that an indirect arrival before definite referral to our centers was associated with a higher probability of in-hospital death. Older age was associated with a 5-fold increased probability of early arrival, possibly due to increased stroke severity in elderly individuals and a known history of vascular risk factors ${ }^{20,21}$. Seeking medical evaluation in the presence of an acute focal neurological deficit is usually fast, even when those manifestations are not necessarily identified as caused by an acute stroke ${ }^{1,2,12}$. Validated pre-hospital clinical rules already exist ${ }^{16,17,22-24}$, and our study did not aim to create new ones. The features included in this analysis were those declared by a bystander witnessing the event and not the ones registered by the healthcare personnel. History of atrial fibrillation could have influenced the case fatality rate through a putative a cardioembolic stroke, a mechanism usually associated with larger infarctions, increased hemorrhagic transformation, and higher recurrence ${ }^{25,26}$. Moreover, atrial fibrillation is also associated with higher mortality after acute hemorrhagic stroke types, and not only in $\mathrm{AlS}^{25}$. According to the present analysis, the signs and symptoms referred by the person witnessing the event had a low PPV for correctly predicting the type of stroke. CPRs based on manifestations and known risk factors can be useful to increase the clinical suspicion of an acute stroke ${ }^{7,24}$. However, their role in distinguishing between stroke subtypes is questionable, especially in distinguishing between AIS and $\mathrm{ICH}$ due to overlapping clinical presentation. Some clinical features and rules could potentially be used to exclude specific neurovascular syndromes due to high sensitivity and NPV (e.g., "language disorder + motor deficit") to identify AIS. In the case of ICH, the correct prediction in excluding other acute neurovascular syndromes was not possible, but the absence of headache by itself could exclude $97 \%$ cases with $\mathrm{SAH}$, which may help in leading the diagnostic approach in this subgroup of patients. Our results cannot endorse the use of clinical rules to accurately pre-classify or determine a stroke subtype to initiate out-of-hospital specialized treatment.

This study has limitations that should be considered to interpret our results correctly. The sample size can be small to identify minor differences in prevalence and outcomes and did not include patients without stroke to compare it among stroke mimics, which is essential to generate mechanisms for fast and efficient dispatch of healthcare attention accurately. A prospective multicenter study with a larger sample, involving the EMS networks and hospitals is needed to prove our hypothesis. It is essential to highlight that our study was not aimed to create diagnostic scores for its employment by the paramedic personnel or in the emergency room, for which validated models exist ${ }^{16,17,22-24}$ since the features included in this analysis are those declared by the patient's bystander and not those registered after a formal physical examination performed by 
healthcare professionals. The fundamental objective of the present study was to investigate the feasibility and impact of a pre-classification of neurovascular syndromes based on witnesses reference to alert the ambulance dispatch personnel to refer the patient directly an in a timely fashion to a prepared hospital that runs organized stroke care. Nevertheless, although the performance of CPRs based on bystanders reference on patients' symptoms seem to be poor in distinguishing among the three main acute neurovascular syndromes, our study provides important data to construct a dedicated pre-hospital stroke dispatching system. First, we confirm that the patient's family or bystanders react usually fast in seeking for medical assessment, but that this assessment occurs in a center that ultimately transfers the patients to a referral hospital. Moreover, we show that pre-classification CPRs have a good performance in discarding stroke syndromes only, but with poor PPV. This finding suggests that recognition of any acute neurological deficit is more relevant than differentiating among stroke syndromes in a pre-hospital setting. A future line of research would be to assess the impact of predicting AIS subtypes by distinguishing lacunar, large-artery, cardioembolic strokes, and other non-lacunar infarctions, given that lacunar syndromes are characteristic and are usually associated with a better short-term outcome ${ }^{27}$.

In conclusion, analysis confirms that the witness of an acute stroke usually acts rapidly and that clinical features recognized by the patients' bystanders have low performance in predicting a specific subtype of stroke. However, some clinical features can be used to exclude the main stroke types and to establish a difference among other acute neurovascular syndromes. We confirmed that pre-hospital factors, such as the type of arrival, age, and level of consciousness, have a negative impact on in-hospital mortality.

\section{SUPPLEMENTARY DATA}

Supplementary data are available at Revista de Investigación Clínica online (www.clinicalandtranslationalinvestigation.com). These data are provided by the corresponding author and published online for the benefit of the reader. The contents of supplementary data are the sole responsibility of the authors.

\section{REFERENCES}

1. Ruiz RG, Fernández JS, Ruiz RM, Bermejo MR, Arias ÁA, Del Saz Saucedo P, et al. Response to symptoms and prehospital delay in stroke patients. Is it time to reconsider stroke awareness campaigns? J Stroke Cerebrovasc Dis. 2018; 27:625-32.

2. Nor AM, McAllister C, Louw SJ, Dyker AG, Davis M, Jenkinson $D$, et al. Agreement between ambulance paramedic-and physician-recorded neurological signs with face arm speech test (FAST) in acute stroke patients. Stroke. 2004;35:1355-9.

3. Gonzalez-Aquines $A$, Cordero-Pérez AC, Cristobal-Niño $M$, Pérez-Vázquez G, Góngora-Rivera F, GECEN Investigators. Contribution of onset-to-alarm time to prehospital delay in patients with ischemic stroke. J Stroke Cerebrovasc Dis. 2019 28:104331.

4. Olascoaga-Arrate A, Freijo-Guerrero MM, Fernandez-Maiztegi C, Azkune-Calle I, Silvariño-Fernández R, Fernández-Rodríguez $M$, et al. Relationship between level of education and one-year survival after ischaemic stroke. Rev Neurol. 2019; 68:147-54

5. Svensson L, Karlsson T, Nordlander R, Wahlin M, Zedigh C, Herlitz J. Implementation of prehospital thrombolysis in Sweden: components of delay until delivery of treatment and examination of treatment feasibility. Int J Cardiol. 2003;88 247-56.

6. Yap YG, Duong T, Bland JM, Malik M, Torp-Pedersen C, Køber L, et al. Prognostic impact of demographic factors and clinical features on the mode of death in high-risk patients after myocardial infarction--a combined analysis from multicenter trials. Clin Cardiol. 2005;28:471-8.

7. Powers WJ, Rabinstein AA, Ackerson T, Adeoye OM, Bambakidis NC, Becker K, et al. Guidelines for the early management of patients with acute ischemic stroke: 2019 update to the 2018 guidelines for the early management of acute ischemic stroke: a guideline for healthcare professionals from the American heart association/American stroke association. Stroke. 2019; 50:e344-418.

8. Fraticelli L, Freyssenge J, Claustre C, Buisson M, Bischoff M, Nighoghossian $N$, et al. Sex-related differences in management and outcome of acute ischemic stroke in eligible patients to thrombolysis. Cerebrovasc Dis. 2019;47:196-204.

9. Wahlgren N, Ahmed N, Dávalos A, Hacke W, Millán M, Muir K, et al. Thrombolysis with alteplase 3-4.5 h after acute ischaemic stroke (SITS-ISTR): an observational study. Lancet. 2008; 372:1303-9

10. León-Jiménez C, Ruiz-Sandoval JL, Chiquete E, Vega-Arroyo M, Arauz A, Murillo-Bonilla LM, et al. Hospital arrival time and functional outcome after acute ischaemic stroke: results from the PREMIER study. Neurologia. 2014;29:200-9.

11. Cheung RT. Hong Kong patients' knowledge of stroke does not influence time-to-hospital presentation. J Clin Neurosci. 2001;8:311-4.

12. Wilhelm LO, Gellert P, White M, Araujo-Soares V, Ford GA, Mackintosh JE, et al. The Recognition-response gap in acute stroke: examining the relationship between stroke recognition and response in a general population survey. J Stroke Cerebrovasc Dis. 2020;29:104499.

13. Goldstein LB, Silberberg M, McMiller Y, Yaggy SD. Stroke-related knowledge among uninsured Latino immigrants in Durham county, North Carolina. J Stroke Cerebrovasc Dis. 2009;18: 229-31.

14. Góngora-Rivera F, González-Aquines A, Muruet W, BarreraBarrera S, Leal-Bailey H, Espinosa-Ortega MA, et al. Difference in stroke knowledge between rural and urban communities in a developing country after community-based stroke educational campaigns: results from a cross-sectional study. Neuroepidemiology 2018:51:224-9

15. Suzuki J, Nakai N, Kondo N, Tsuji H, Inagaki R, Furukawa S, et al. Ten-year evaluation of the TOYOTA prehospital stroke scale for tissue plasminogen activator intravenous therapy in the real world. Cerebrovasc Dis. 2018;46:184-92

16. Kidwell CS, Saver JL, Schubert GB, Eckstein M, Starkman S. Design and retrospective analysis of the Los Angeles prehospital stroke screen (LAPSS). Prehospital Emerg Care. 1998; 2:267-73.

17. Vidale S, Agostoni E. Prehospital stroke scales and large vessel occlusion: a systematic review. Acta Neurol Scand. 2018; 138:24-31. 
18. Langhorne P, Ramachandra S, Stroke Unit Trialists' Collaboration. Organised inpatient (stroke unit) care for stroke: network meta-analysis. Cochrane Database Syst Rev. 2020;4:CD000197.

19. Ruiz-Sandoval JL, González-García C, García-Navarro V, Gutiérrez-Monjarrez FA, González-Cornejo S. Enfermedad vascular cerebral isquemica en dos hospitales mexicanos. Rev Mex Neuroci. 2003;4:319-23.

20. Morey JR, Dangayach NS, Shoirah H, Scaggiante J, Mocco J, Tuhrim S, et al. Major causes for not performing endovascular therapy following inter-hospital transfer in a complex urban setting. Cerebrovasc Dis. 2019;48:109-14.

21. Chiquete E, Torres-Octavo B, Cano-Nigenda V, Valle-Rojas $D$, Dominguez-Moreno R, Tolosa-Tort $P$, et al. Characterisation of factors associated with carotid stenosis in a population at high risk. Rev Neurol. 2014;58:541-7.

22. Kothari RU, Pancioli A, Liu T, Brott T, Broderick J. Cincinnati prehospital stroke scale: reproducibility and validity. Ann Emerg Med. 1999;33:373-8.
23. Nor AM, Davis ], Sen B, Shipsey D, Louw SJ, Dyker AG, et al. The recognition of stroke in the emergency room (ROSIER) scale: development and validation of a stroke recognition instrument. Lancet Neurol. 2005:4:727-34.

24. Uchida K, Yoshimura S, Hiyama N, Oki Y, Matsumoto T, Tokuda $\mathrm{R}$, et al. Clinical prediction rules to classify types of stroke at prehospital stage: Japan urgent stroke triage (JUST) score. Stroke. 2018;49:1820-7.

25. Arboix A, Alió J. Acute cardioembolic stroke: an update. Expert Rev Cardiovasc Ther. 2011;9:367-79.

26. Griñán K, Arboix A, Massons J, Díez L, Vergés E, Gil F, et al. Cardioembolic stroke: risk factors, clinical features, and early outcome in 956 consecutive patients. Rev Invest Clin. 2020 [Epub ahead of print].

27. Arboix A, Blanco-Rojas L, Martí-Vilalta JL. Advancements in understanding the mechanisms of symptomatic lacunar ischemic stroke: translation of knowledge to prevention strategies. Expert Rev Neurother. 2014;14:261-76. 